\title{
Evaluation of cloud brokering algorithms in cloud based data center
}

\begin{abstract}
Migrating to the cloud for the purpose of minimizing infrastructure costs and technical effort is a common trend these days. Over the last couple of years, we have witnessed an increasing demand for cloud computing, which lead to the increase of both cloud users, and cloud service providers as well. Due to the chaos of the various service providers, it is very difficult for a user to choose the appropriate provider that satisfies their specific requirements. A new research paradigm has emerged in an attempt to address this problem. Cloud interoperability is another issue that allows users to easily migrate applications and workloads across cloud service providers. The definition of a common standard between cloud providers has the ability of eliminating this issue. Another approach is the use of a cloud service broker, which assists users to find appropriate cloud service providers before the deployment of their application or service. A broker is capable of finding an appropriate service provider that would satisfy user service requirements in terms of a service level agreement. In this paper, two new cloud brokering algorithms, and their initial evaluation, are proposed.
\end{abstract}

Keyword: Cloud brokering; Brokering algorithm; Cloud simulation; Load balancing; Interoperability 